\title{
Analysis of Dual-Reflector Antennas with a Reflectarray as Subreflector
}

\author{
Manuel Arrebola ${ }^{1}$, Leandro de Haro', and José A. Encinar ${ }^{3}$ \\ ${ }^{1}$ Dpto. de Ingenieria Eléctrica, Área de Teoria de la Señal y Comunicaciones \\ Universidad de Oviedo \\ Edificio Polivalente de Viesques (8.1.13), Campus de Universitario de Gijón s/n, E-33203 Gijón, Spain \\ E-mail: arrebola@tsc.uniovi.es \\ ${ }^{2}$ Dpto. de Señales, Sistemas y Radiocomunicaciones, Grupo de Radiación \\ Universidad Politécnica de Madrid \\ ETSI Telecomunicación, Ciudad Universitaria s/n, E-28040 Madrid, Spain \\ E-mail: leandro.deharo.ariet@upm.es \\ ${ }^{3}$ Dpto. de Electromagnetismo y Teoría de Circuitos \\ Universidad Politécnica de Madrid \\ ETSI Telecomunicación (B-414), Ciudad Universitaria s/n, E-28040 Madrid, Spain \\ E-mail: jose.encinar@upm.es
}

\begin{abstract}
In this paper, a modular technique is described for the analysis of dual-reflector antennas using a reflectarray as a subreflector. An antenna configuration based on a sub-reflectarray and a parabolic main reflector provides better bandwidth than a single reflectarray, and has a number of advantages compared with a conventional dual-reflector antenna. Examples include the possibility of beam shaping by adjusting the phase on the sub-reflectarray, and potential capabilities to scan or reconfigure the beam. The modular technique implemented for the antenna analysis combines different methods for the analysis of each part of the antenna. First, the real field generated by the horn is considered as the incident field on each reflectarray element. Second, the reflectarray is analyzed with the same technique as for a single reflectarray, i.e., considering local periodicity and the real angle of incidence of the wave coming from the feed for each periodic cell. Third, the main reflector is analyzed using the Physical Optics (PO) technique, where the current on the reflector surface is calculated by summing the radiation from all the reflectarray elements. Finally, the field is calculated on a rectangular periodic mesh at a projected aperture, and then a time-efficient fast Fourier transform (FFT) algorithm is used to compute the radiation pattern of the antenna. The last step significantly improves the computational efficiency. However, it introduces a phase error, which reduces the accuracy of the radiation patterns for radiation angles far away from the antenna's axis. The phase errors have been evaluated for two integration apertures. It has been demonstrated that accurate patterns are obtained in an angular range of $\pm 6^{\circ}$, which is sufficient for large reflectors. The method of analysis has been validated by comparing the results with simulations obtained from GRASP8. Finally, the theoretical beam-scanning performance of the antenna is analyzed.
\end{abstract}

Keywords: Multireflector antennas; reflector antennas; reflectarray; shaped beam antennas; beam steering

\section{Introduction}

$\mathbf{P}$ rinted reflectarray antennas can be alternatives to classic reflectors in many applications. They have a number of advantages, such as low profile, mass, and volume; an easy manufacturing process; and possibilities for beam shaping and electric beam control $[1,2]$. A printed reflectarray is a planar array of printed elements illuminated by a primary feed, typically a horn antenna. Each element of the reflectarray introduces a phase shift to the wave from the feed impinging on the reflectarray, so that pencil or contoured beams are obtained. A number of works have been published on the design of reflectarrays in different configu- rations and for different applications. Contoured-beam reflectarrays are especially interesting, and have been demonstrated using patches of variable size in single-layer [3] and multilayer configurations for bandwidth improvement $[4,5]$. Reconfigurable and active reflectarrays have also been demonstrated using different technologies $[6,7]$. Although the typical configuration is a single reflectarray, folded reflectarrays have been proposed for radar applications [8].

The main limitation of reflectarrays is their narrow bandwidth. This is primarily caused by two factors: the bandwidth of the microstrip element, and the differential spatial phase delay $[9$, 10]. The element bandwidth, which does not depend on the size of 
the reflectarray, can be improved through appropriate design of the phase shifter, for example by using two or three stacked layers with patches of varying size $[4,11]$. Elements with delay lines can also be introduced into the design of the reflectarray [12]. Recently, an artificial-impedance-surface concept was proposed to increase the bandwidth [13]. The second limitation for reflectarray bandwidth is more important in electrically large reflectarrays $[9,10]$, where the differential spatial phase delay is much larger than for small reflectarrays. As a result, electrically small reflectarrays are preferred. The smaller dimensions simplify the manufacturing processes, and also allow possible implementation of electrical beam control by adding a controllable phase shifter at the reflectarray elements.

On the other hand, classical parabolic reflectors have a theoretically infinite bandwidth because the beam is focused by the parabolic surface independently of the frequency [14]. The manufacturing process is well known, but it is also complex and expensive for shaped reflectors. Another disadvantage is that the design of a reconfigurable reflector antenna is based on mechanical devices or feed-horn clusters, which are heavy and mass- and volume-consuming solutions. Dual-reflector configurations are used to improve some aspects of the performance of single reflectors, such as cross-polar radiation, or to reduce the volume of the whole antenna.

As in the case of single reflectors, reflectarrays can be also an alternative in dual-reflector structures. In a general dual-reflector antenna, a reflectarray can be used either as a subreflector or as the main reflector [15]. In fact, both of these can be reflectarrays, as in the folded configuration described in [16]. Recently, a dual-reflector antenna with a reflectarray as the subreflector was proposed for compensating the errors on the surface of very large deployable reflectarrays [17].

The sub-reflectarray/parabolic main reflector configuration provides better bandwidth than a single reflectarray antenna because of the reduced electrical dimensions of the reflectarray, as mentioned above. In addition, this dual-reflector configuration is very appropriate for electronic beam agility, because the beam can be scanned or reconfigured by controlling the phase at the elements of a reduced-size reflectarray. In particular, there are two space applications than can benefit from the proposed antenna configuration with beam-scanning capabilities. The first application is synthetic-aperture radar (SAR). This is usually implemented using active arrays, but a reduction in cost and complexity is desirable. An X-band SAR using a passive reflector antenna was recently developed (SAR_Lupe) [18], but SAR antennas providing beam scanning at a moderate cost are still a main concern. A second application is in radiometric remote-sensing missions. In the $300 \mathrm{GHz}$ band, reflector antennas with mechanical mechanisms are used to provide the beam scanning in this type of mission [19]. An alternative for electronic beam scanning in these frequency bands can be reflectarrays based on liquid crystals [20,21]. An steerablebeam reflectarray was demonstrated in [21] at $34 \mathrm{GHz}$, but the same concept can be used at higher frequencies [22]. The use of a dual-reflector configuration with liquid crystals on only a small sub-reflectarray would simplify the manufacturing process and the temperature control in a space environment.

This dual-reflector configuration can be also used in shapedbeam applications, such as DBS (direct-broadcast satellite) missions. In this case, the contoured beam can be obtained by synthe- sizing the phase distribution on the flat reflectarray used as a subreflector, while the main parabolic reflector is used to focus the beam with no limitation on the frequency band. This geometry could thus exhibit better bandwidth behavior than a single reflectarray, and it is simpler to manufacture than a shaped reflector.

The proposed antenna configuration cannot be analyzed using available commercial tools. Some approximate techniques have been proposed for the analysis of dual-reflector antennas involving reflectarrays, but an analysis technique for a general sub-reflectarray/main reflector antenna has not been described in the literature up until now.

A dual-reflectarray antenna in folded configuration was proposed in [16]. In this configuration, the feed horn is embedded in the center of the main reflectarray. The horn illuminates a subreflectarray based on varying-sized dipoles printed on a substrate layer backed by a strip grating, which acts as a ground plane for the polarization of the feed. The field reflected by the first reflectarray impinges on the main reflectarray, where it is focused and also twisted $90^{\circ}$ by the main reflectarray, so that the reflected field can pass trough the strip grating containing the first reflectarray. The antenna was designed using a technique based on ray tracing, and the reflectarray elements were analyzed by the Method of Moments (MoM). However, the technique is only applicable to this particular "folded" configuration, which is only valid for linear polarization.

A dual-reflector configuration using a reflectarray as a subreflector was analyzed in [17]. In this case, the analysis of the reflector was based on Physical Optics, but the characterization of the sub-reflectarray was based on a simple design curve validated by measurements of the element in a waveguide simulator. In this approach, neither the cross-polarization generated by the printed elements nor the angle of incidence of the impinging wave on each reflectarray element are taken into account in the analysis of the reflectarray. In a dual-reflector antenna, the subreflector is normally illuminated in the near-field region of the primary feed, where the angles of incidence are moderately wide, and therefore they should be considered in the analysis of the sub-reflectarray.

In this paper, a modular technique is proposed for the analysis of the dual-reflector antenna made up of a sub-reflectarray and a parabolic reflector. The analysis technique is based on the combination of different methods for the analysis of each component of the antenna. The primary feed, which typically is a horn antenna, can be analyzed either using a full-wave method, or modeled using a far-field approximation, so that the incident field on the subreflectarray is calculated. The sub-reflectarray is analyzed as a single reflectarray through the Method of Moments (MoM) considering local periodicity, and the parabolic reflector is analyzed using Physical Optics (PO). Finally, the radiation pattern is calculated with a time-efficient fast Fourier transform (FFT) algorithm from the aperture field projected onto a planar surface.

The analysis technique is described in Section 2 and validated in Section 3. For the validation, a simple reflectarray that introduces a uniform phase distribution and compensates for the effect of the angle of incidence has been designed. The results are compared with those obtained by GRASP-8.1 when a metallic subreflector is considered. Finally, as an example of an application, the theoretical beam-scanning capabilities of the antenna are studied in Section 4. 


\section{Analysis Technique}

A general schematic of the proposed configuration is shown in Figure 1. The antenna is made up of three elements: a primary feed and two reflectors, a sub-reflectarray, and a parabolic reflector. In this work, an offset configuration is considered, but the analysis technique is general and can be applied to centered geometries.

In principle, a horn antenna is considered as the primary feed, but the subreflector can be illuminated by another structure, such as an array or a cluster of horns. The subreflector is a multilayer reflectarray of printed stacked patches of varying size above a ground plane. However, other elements, different from stacked patches, can be used for the reflectarray, such as dipoles, slots, or rings. The main reflector is defined as parabolic, so that the structure can be considered to be a Newtonian antenna, with an adjustment on the phase of the reflectarray acting as subreflector.

Three coordinate systems are used in the analysis process: the feed reference system, $\left(\mathbf{x}_{F}, \mathbf{y}_{F}, \mathbf{z}_{F}\right)$, which is centered at the phase center of the primary feed; the reflectarray (subreflector) reference system, defined by $\left(\mathbf{x}_{R}, \mathbf{y}_{R}, \mathbf{z}_{R}\right)$, the origin of which is in the center of the reflectarray surface; and the antenna reference system, $\left(\mathbf{x}_{A}, \mathbf{y}_{A}, \mathbf{z}_{A}\right)$.

The proposed analysis method is a modular approach that combines the Method of Moments (MoM) for the analysis of the sub-reflectarray and Physical Optics (PO) for the main parabolic reflector. The method provides good accuracy and time efficiency, so it can be used in an optimization process. In the following subsections, the analysis techniques for the different components of the antenna, feed horn, sub-reflectarray, and parabolic reflector are detailed.

\subsection{Feed Horn}

In dual-reflector geometries, the subreflector is typically placed in the near-field region of the feed horn. The actual incident field should be taken into account for an accurate analysis of the sub-reflectarray [23]. In this case, the near field at the center of each reflectarray element can be obtained from simulations or measurements. The components of the field are typically obtained in the feed reference system, so that a transformation matrix $(\mathbf{A})$ is used to transform the components into the reflectarray reference system (see Figure 1 and Equation (1)):

$$
\mathbf{E}_{\text {inc } R}^{X / Y}=E_{\text {xinc } R}^{X / Y} \mathbf{x}_{R}+E_{y \text { inc } R}^{X / Y} \mathbf{y}_{R}=\mathbf{A} \cdot \mathbf{E}_{\text {inc } F}^{X / Y} .
$$

Since two linear polarizations, $\mathbf{x}_{A}$ and $\mathbf{y}_{A}$, can be considered, the $\mathrm{X} / \mathrm{Y}$ superscript denotes the polarization defined by the feed. For example, $\mathbf{E}_{\text {incF }}^{X}$ is the electric field on a reflectarray element produced by a feed with the electric field polarized in the $\mathbf{x}_{F}$ direction. Note that this field contains both $x$ and $y$ components in the reflectarray coordinate system. The near field radiated by the feed on each reflectarray element can be obtained by direct near-field measurements, or by source reconstruction using far-field measurements of the horn, as described in [24]. In some cases, a farfield model of the feed horn can be a valid approximation in the Fresnel zone, but it must be checked for each geometry [24].

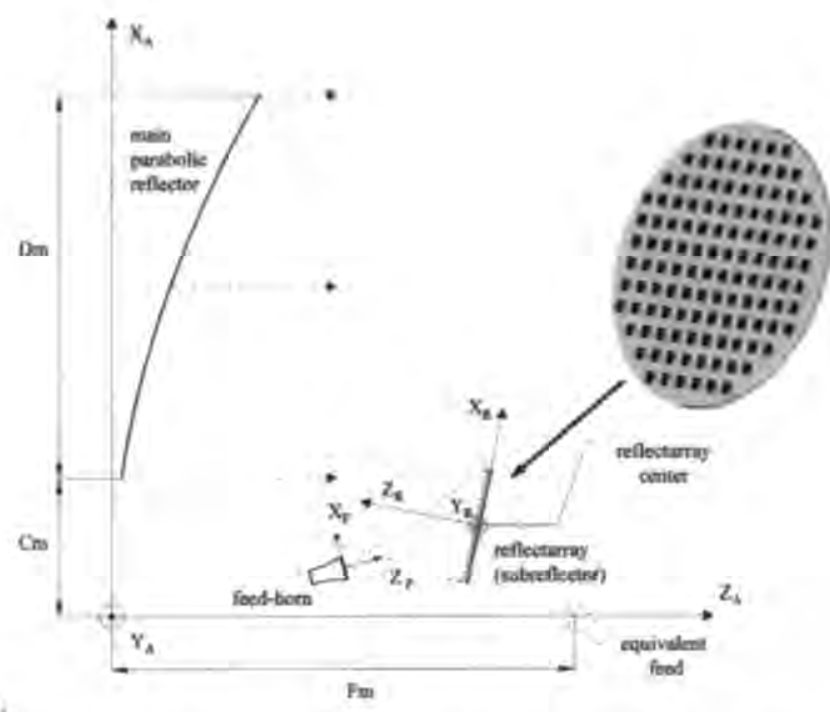

Figure 1. The schematic of a sub-reflectarray/parabolic-reflector structure.

\subsection{Sub-Reflectarray}

The subreflector is a flat multilayer reflectarray of printed stacked patches, which is analyzed using the same technique as in a single reflectarray, as explained in [4]. The analysis is carried out by computing the total reflected field on each cell through a fullwave analysis based on the Method of Moments (MoM) in the spectral domain, assuming each element is in a periodic environment [25]. The total field reflected by the reflectarray elements is computed by MoM as the superposition of the field reradiated by the patches and the field reflected by the multilayer dielectric structure (when the printed patches are not present). Note that the last term is not present in phased arrays where the radiating elements are fed individually, but it should be included in reflectarrays. The calculation of the reflected field includes the crosspolarization components produced by the reflectarray elements and by the field transformations from the feed to the reflectarray coordinate system, as shown in Equation (1). The total reflected field on each $m n$th cell can be written as

$$
\mathbf{E}_{\text {ref }}^{X / Y}\left(m_{R}, n_{R}\right)=\mathbf{R}\left(m_{R}, n_{R}\right) \cdot \mathbf{E}_{\text {incR }}^{X / Y}\left(m_{R}, n_{R}\right),
$$

where

$$
\mathbf{R}=\left(\begin{array}{ll}
\rho_{x x} & \rho_{y x} \\
\rho_{x y} & \rho_{y y}
\end{array}\right) .
$$

The components of the $\mathbf{R}$ matrix are complex reflection coefficients defined for the total reflected field, and they characterize the behavior of the reflectarray element. The components $\rho_{x x}$ and $\rho_{y x}$ are the direct and cross reflection coefficients for an incident wave polarized with the electric field in the $\mathbf{x}_{R}$ direction (without a component in $\mathbf{y}_{R}$ ). On the other hand, $\rho_{y y}$ and $\rho_{x y}$ are the reflection coefficients for an incident electric field in the $\mathbf{y}_{R}$ direction. They depend on the angle of incidence of the impinging wave coming from the feed, so that the matrix is computed for each element of the reflectarray. 


\subsection{Main Reflector}

The analysis of the main reflector is based on Physical Optics (PO) techniques. The electrical current on the reflector surface can be computed in terms of the normal vector, $\mathbf{n}$, and the incident magnetic field, $\mathbf{H}_{\text {parab }}$, as

$$
\mathbf{J}^{X / Y}=2 \mathbf{n} \times \mathbf{H}_{\text {in parab }}^{X / Y}
$$

The incident magnetic field is computed by adding the contributions of the field radiated by each reflectarray element $\left(m_{R}, n_{R}\right)$ :

$$
\mathbf{H}_{\text {in parab }}^{X / Y}=\sum_{m_{R}} \sum_{n_{R}} \mathbf{H}_{i n}^{X / Y}\left(m_{R}, n_{R}\right)
$$

Each element $\left(m_{R}, n_{R}\right)$ of the reflectarray is modeled as a rectangular aperture of dimension $a \times b$ with the electric field constant, so that the radiated field is obtained as

$$
\begin{aligned}
& H_{\theta \text { in }}^{X / Y}\left(m_{R}, n_{R}\right) \\
& =M\left\{\cos \theta\left[E_{x r e f}^{X / Y}\left(m_{R}, n_{R}\right) \sin \phi-E_{y r e f}^{X / Y}\left(m_{R}, n_{R}\right) \cos \phi\right]\right\}, \\
& H_{\phi \text { in }}^{X / Y}\left(m_{R}, n_{R}\right)=M\left[E_{x \text { ref }}^{X / Y}\left(m_{R}, n_{R}\right) \cos \phi+E_{y \text { ref }}^{X / Y}\left(m_{R}, n_{R}\right) \sin \phi\right],
\end{aligned}
$$

where

$$
M=j k_{0} \frac{\exp \left(-j k_{0} r\right)}{2 \pi \eta_{0} r} a b \operatorname{sinc}\left(\frac{k_{u} a}{2}\right) \operatorname{sinc}\left(\frac{k_{v} b}{2}\right)
$$

and

$$
\begin{aligned}
& k_{u}=k_{0} u=k_{0} \sin \theta \cos \phi, \\
& k_{v}=k_{0} v=k_{0} \sin \theta \sin \phi .
\end{aligned}
$$

Once the PO electric current distribution, Equation (4), is known, the field on the projected aperture plane is obtained by means of

$$
\mathbf{E}_{a p}^{X / Y}=\left[\mathbf{n}_{a p} \times\left(\mathbf{n}_{a p} \times \mathbf{J}^{X / Y}\right)\right] \mathrm{Jac} \exp (j \varphi),
$$

where Jac is the Jacobian transformation of the current on the aperture plane, $\mathbf{n}_{a p}$ is the vector normal to the aperture considered, and the last term is the phase factor introduced because of the displacement from the reflector to the integration plane [26]:

$$
\varphi=k_{0}\left(z-z_{a p}\right)
$$

The phase factor is a simple approximation that is applied to allow fast computation of the secondary radiation field. It simplifies the computation but includes a phase error on the aperture, which limits the minimum field value of computed sidelobes. The limitations introduced by this approximation are discussed in Section 3.1 .

\subsection{Integration of the Field at the Aperture}

Once the field at the aperture has been calculated, the radiated field is computed. Assuming the First Principle of Equivalence, the components $\theta$ and $\phi$ of the radiated far field are given by

$$
\begin{aligned}
E_{\theta}^{X / Y}(u, v)=j k_{0} \exp \left(-j k_{0} r\right)(1+\cos \theta) & \\
& {\left[P_{x}^{X / Y}(u, v) \cos \phi+P_{y}^{X / Y}(u, v) \sin \phi\right] / 4 \pi r } \\
E_{\phi}^{X / Y}(u, v)=- & j k_{0} \exp \left(-j k_{0} r\right)(1+\cos \theta) \\
& {\left[P_{x}^{X / Y}(u, v) \sin \phi-P_{y}^{X / Y}(u, v) \cos \phi\right] / 4 \pi r }
\end{aligned}
$$

where $u$ and $v$ are defined in Equation (9), and $P_{x}^{X / Y}(u, v)$ and $P_{y}^{X / Y}(u, v)$ are the radiation integrals throughout the aperture surface, $A P$ :

$$
P_{x}^{X / Y}(u, v)=\iint_{A P} E_{x a p}^{X / Y}\left(x_{A}, y_{A}\right) \exp \left[j k_{0}\left(u x_{A}+v y_{A}\right)\right] d x_{A} d y_{A},
$$

$$
P_{y}^{X / Y}(u, v)=\iint_{A P} E_{y a p}^{X / Y}\left(x_{A}, y_{A}\right) \exp \left[j k_{0}\left(u x_{A}+v y_{A}\right)\right] d x_{A} d y_{A}
$$

The field has been calculated at the aperture by considering a regular mesh of period $\Delta x$ and $\Delta y$ in the $x_{A}$ and $y_{A}$ directions, respectively. If the field is assumed to be constant in each cell $\left(m_{A}, n_{A}\right)$ of the grid, the double integral of Equation (14) can be written as a double sum, as follows:

$$
P_{x}^{X / Y}(u, v)=K \sum_{m_{A}} \sum_{n_{A}} E_{x a p}^{X / Y}\left(m_{A}, n_{A}\right) \exp \left[j k_{0}\left(u m_{A} \Delta x+v n_{A} \Delta y\right)\right],
$$

$$
P_{y}^{X / Y}(u, v)=K \sum_{m_{A}} \sum_{n_{A}} E_{y a p}^{X / Y}\left(m_{A}, n_{A}\right) \exp \left[j k_{0}\left(u m_{A} \Delta x+v n_{A} \Delta y\right)\right],
$$

where $E_{a p}^{X / Y}\left(m_{A}, n_{A}\right)$ is the complex field at the cell $\left(m_{A}, n_{A}\right)$ of the grid, and $K$ is

$$
K=\Delta x \Delta y \operatorname{sinc}\left(k_{0} u \Delta x / 2\right) \operatorname{sinc}\left(k_{0} v \Delta y / 2\right) .
$$

In Equation (15), the double sum can be expressed as a discrete Fourier transform, so that $P_{x}^{X / Y}$ and $P_{y}^{X / Y}$ are calculated as

$$
\begin{aligned}
& P_{x}^{X / Y}(p, q)=N_{x} N_{y} K \operatorname{IDFT} 2\left[E_{x a p}^{X / Y}\left(m_{A}, n_{A}\right)\right] \\
& P_{y}^{X / Y}(p, q)=N_{x} N_{y} K \operatorname{IDFT} 2\left[E_{y a p}^{X / Y}\left(m_{A}, n_{A}\right)\right]
\end{aligned}
$$


where IDFT2 is the two-dimensional inverse discrete Fourier transform. The indices $p$ and $q$ are related to $u$ and $v$ by

$$
\begin{aligned}
& u=2 \pi p / N_{x} \Delta x k_{0}, \\
& v=2 \pi q / N_{y} \Delta y k_{0} .
\end{aligned}
$$

Finally, the IDFT2 function is implemented by a time-efficient two-dimensional fast Fourier transform (FFT) algorithm so that the radiation pattern is computed in all the space at the same time, not point-by-point. This technique is especially useful in the calculation of three-dimensional radiation patterns.

\section{Technique Validation}

Two studies have been carried out in order to validate the proposed analysis technique. First, the phase error introduced by Equation (11) was calculated, so that the accuracy for different angles in the radiation pattern was evaluated. Second, the proposed technique was applied to the analysis of a dual-reflector antenna with a reflectarray as a subreflector, considering the cases of an ideal and a real reflectarray.

The antenna was made up of a feed horn, a planar reflectarray as the subreflector, and a parabolic metallic surface as the main reflector, with the geometrical data summarized in Table 1. The spillover was computed for the sub-reflectarray and for the main reflector with respect to the total power radiated by the horn. The feed horn was oriented towards a point on the sub-reflectarray's surface (pointing of the horn) given in Table 1, which was set to minimize the spillover produced by the subreflector. The projected aperture for the main reflector (see Figure 2) was circular, and the sub-reflectarray was defined as rectangular. In this case, the primary feed was a horn antenna modeled as a $\cos ^{q}(\theta)$ function, and the $q$ factor was chosen so that the illumination at the edges of the sub-reflectarray was about $-18 \mathrm{~dB}$. The selected frequency was $11.95 \mathrm{GHz}$, and the polarization considered was vertical ( $X_{A}$ pol.) for all the cases. The size of the reflectarray aperture was $20 \lambda \times 20 \lambda$, which could be considered small for local-periodicitybased analysis, particularly when illuminated in the Fresnel zone of the feed. However, good accuracy of the method was demonstrated in previous work using smaller reflectarrays, e.g., the reflectarray designed, manufactured, and measured in [27] was $15 \lambda \times 15 \lambda$ and was illuminated in the near-field zone of the feed horn. The results shown in that reference showed good agreement between simulations and measurements. In the analysis, the blockage and diffraction at the edges was not considered. Note that from a practical standpoint, the reflectarray elements near the edge do not contribute to the radiation patterns because of the very low illumination $(-18 \mathrm{~dB})$.

\subsection{Angular Validation: Phase Error at the Aperture Plane}

The proposed analysis technique was detailed in Section 2. In order to calculate the radiation patterns, the approximate phase factor given by Equation (11) was used to project the PO-printed currents on the parabolic surface onto the planar aperture. However, the analysis technique for the entire antenna is not based on ray tracing. This approximation reduces the computational time but
Table 1. The main data for the geometry studied.

\begin{tabular}{|c|c|}
\hline Diameter of projected aperture (Dm) & $1.5 \mathrm{~m}$ \\
\hline Clearance $(\mathrm{Cm})$ & $0.2 \mathrm{~m}$ \\
\hline Focal distance $(\mathrm{Fm})$ & $1.5 \mathrm{~m}$ \\
\hline Spillover & $-25.2 \mathrm{~dB}$ \\
\hline \multicolumn{2}{|c|}{$\begin{array}{c}\text { Sub-Reflectarray } \\
\text { (data in antenna coordinate system) }\end{array}$} \\
\hline Center (xfm, yfm, $\mathrm{zfm})$ & $(0.294,0.0,1.174) \mathrm{m}$ \\
\hline Sub-reflectarray dimensions & $520 \times 494 \mathrm{~mm}$ \\
\hline $\begin{array}{l}\text { Direction cosines matrix (CosDir) } \\
\text { Relation between antenna and subreflector } \\
\text { coordinate systems }\end{array}$ & $\left(\begin{array}{ccc}1 & 0 & 0 \\
0 & -1 & 0 \\
0 & 0 & -1\end{array}\right)$ \\
\hline Spillover & $-20.4 \mathrm{~dB}$ \\
\hline \multicolumn{2}{|c|}{$\begin{array}{c}\text { Feed Horn } \\
\text { (data in subreflector coordinate system) }\end{array}$} \\
\hline Phase center & $(-294,0,326) \mathrm{mm}$ \\
\hline $\begin{array}{l}\text { Pointing of the horn } \\
\text { (on the sub-reflectarray surface) }\end{array}$ & $(-56,0,0) \mathrm{mm}$ \\
\hline $\begin{array}{l}\text { Illumination level at the sub-reflectarray } \\
\text { edges }\end{array}$ & $-18 \mathrm{~dB}$ \\
\hline
\end{tabular}

Main Parabolic Reflector

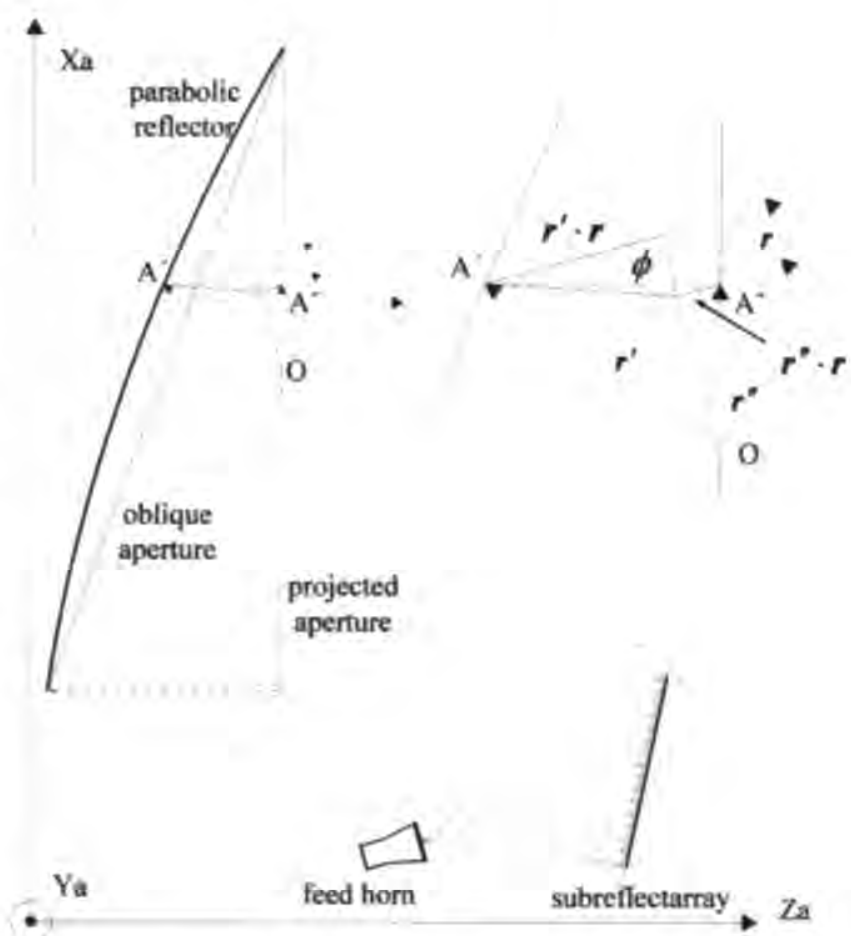

Figure 2. The phase error introduced by the calculation of the radiation pattern starting from the equivalent field at the projected aperture.

also limits the angular range of the analysis technique, because of the introduction of a phase error in the calculation of the field at the aperture. The phase error depends on the aperture used for the integration of the field. Here, two apertures were considered and the results were compared. First, the classic aperture projected on a plane normal to the $z_{A}$ axis was studied. As an alternative, an elliptic oblique aperture, which was defined by the contour of the parabolic reflector, was also considered. The two apertures are shown in Figure 2, and the vectors in the figure refer to the direc- 
tions where the radiation patterns were computed. The aperture coordinate system was defined by $\left(\mathbf{x}_{a p}, \mathbf{y}_{a p}, \mathbf{z}_{a p}\right)$, and its origin, point $\mathrm{O}$, was at the center of the aperture. For the case of the projected aperture, the aperture coordinate system was the same as the antenna coordinate system $\left(\mathbf{x}_{A}, \mathbf{y}_{A}, \mathbf{z}_{A}\right)$ with a displacement of the origin. In the case of the oblique aperture, the $\mathbf{y}_{a p}$ vector was the same as $\mathbf{y}_{A}$, but the $\mathbf{x}_{a p}$ and $\mathbf{z}_{a p}$ vectors were rotated at the same angle as the aperture.

The phase error was caused by the different positions of the current cells at the paraboloid's surface and the equivalent field cells at the aperture, and, consequently, by the different position vectors. Here, the case of the projected aperture is discussed and, therefore, these vectors are $\mathbf{r}^{\prime}$ and $\mathbf{r}^{\prime \prime}$ (see Figure 2). They are the position vectors in the aperture coordinate system of the points $\mathrm{A}^{\prime}$ and $\mathrm{A}^{\prime \prime}$ on the parabolic surface and the projected aperture, respectively.

In the calculation of the radiation pattern produced by a field distribution on a surface, typically an aperture, this field distribution has to be integrated. If the far field is assumed, the exponential term of the radiation integral can be simplified to be the dot product of two vectors: the position vector drawn from the origin to the point of the field distribution considered, and the unit vector drawn from the origin toward the direction where the radiated field is calculated.

The electrical current is usually integrated at the parabolic surface in order to calculate the radiation pattern, so that the actual placement of the current cell is taken into account. In this case, $\mathbf{r}^{\prime}$ is the position vector of the point $\mathrm{A}^{\prime}$ on the parabolic reflector, and $\mathbf{r}$ is the unitary vector given by direction where the radiation pattern is calculated. The exponential term of the radiation term is thus

$$
\exp \left[j k_{0}\left(\mathbf{r}^{\prime} \cdot \mathbf{r}\right)\right]=\exp \left(j p^{\prime}\right)
$$

The $k_{0}\left(\mathbf{r}^{\prime} \cdot \mathbf{r}\right)$ term can be considered a phase term, $p^{\prime}$, which depends on the placement of the $\mathrm{A}^{\prime}$ point and the $\mathbf{r}$ vector.

In the case of the integration at the projected aperture, the equivalent electric field at the aperture has to be calculated. Here, the phase factor, $\phi$, given by Equation (11) has been introduced. The exponential term is the same for the same $\mathbf{r}$ vector, but using the position vector, $\mathbf{r}^{\prime \prime}$ of the point $A^{\prime \prime}$ at the aperture. However, the radiation integral has another exponential term in relation to the previous case because of the phase term, and the overall exponential term will be

$$
\begin{aligned}
\exp \left[j k_{0}\left(\mathbf{r}^{n} \cdot \mathbf{r}\right)\right] \exp \left(j k_{0} \varphi\right) & =\exp \left\{j k_{0}\left[\left(\mathbf{r}^{n} \cdot \mathbf{r}\right)+\varphi\right]\right\} \\
& =\exp \left(j p^{\prime \prime}\right),
\end{aligned}
$$

where $p^{\prime \prime}$ is the overall phase term in the case of the projected aperture. The phase error introduced by the approximation of the field at the projected aperture is

$$
\text { error }=p^{\prime}-p^{\prime \prime}=\mathbf{r}^{\prime} \cdot \mathbf{r}-\left(\mathbf{r}^{\prime \prime} \cdot \mathbf{r}+\varphi\right)
$$

The error can be seen graphically in Figure 2. It depends on the $\mathbf{r}$ vector that defines the direction where the pattern is computed. In order to have no error in the calculation of the radiation pattern at any radiation angle, the phase factor $\phi$ should be $\mathbf{r}^{\prime \prime} \cdot \mathbf{r}-\mathbf{r}^{\prime} \cdot \mathbf{r}$. Note that this phase correction depends on the direction in which the radiated field is computed, and also depends on the overall threedimensional radiation pattern being computed at the same time by the proposed technique. Moreover, this approximation is accurate enough for the small radiation angles that are usually required in large-aperture applications.

In the case of the integration on the oblique aperture, the phase error in the calculation of the equivalent field is less, and thus the analysis is accurate over a wider angle range.

The phase errors were evaluated for the antenna defined in Table 1 for different radiation angles. The working frequency considered was $11.95 \mathrm{GHz}$. Two integration apertures, the projected and oblique apertures as shown in Figure 2, were considered, in order to estimate the angular accuracy. The phase-error maps shown in Figure 3 were calculated supposing a radiation angle of $5^{\circ}$. For this angle, the phase error of the equivalent field on the aperture was larger than $-30^{\circ}$ for some elements considering a projected aperture (see Figure $3 \mathrm{a}$ ). For the case of an oblique aperture and for the same radiation angle, the phase error remained less than $-7^{\circ}$ (see Figure $3 b$ ).

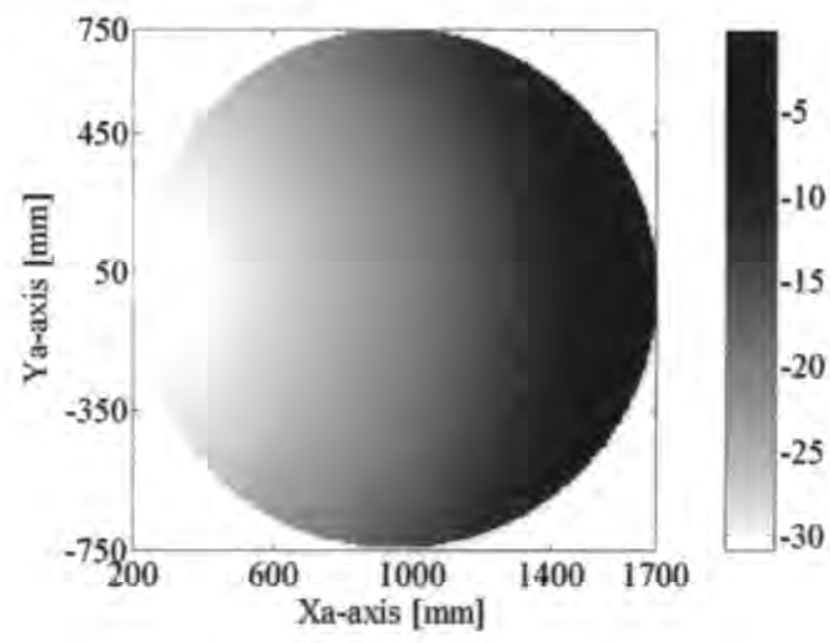

(a)

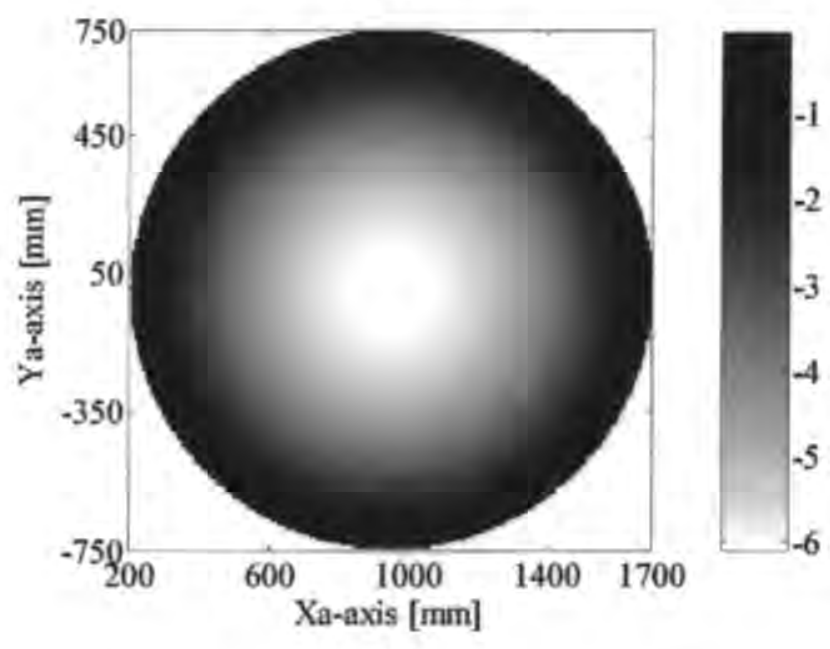

(b)

Figure 3. A phase-error map [deg.] for a $5^{\circ}$ radiating angle at 11.95 GHz: projected aperture (a) and oblique aperture (b). 
In Figure 4, the main vertical cut $\left(\phi=0^{\circ}\right)$ of the phase-error map has been plotted for three radiation angles, considering the two aperture planes. This showed that the field integration at the projected aperture caused much more phase error than at the oblique aperture. As a result, the phase error introduced by Equation (11) limits the valid angular range of the analysis method. The error cannot be corrected, because it depends on the current element and the $(\theta, \phi)$ direction defined by the $\mathbf{r}$ vector, and the entire three-dimensional radiation pattern is computed by the IDFT2 function at the same time. Nevertheless, this kind of antenna is required for high-gain applications, such as space applications, that do not require a wide angular range. In fact, the Earth is covered by $\pm 8.7^{\circ}$ from a geostationary satellite. The azimuth plane $\left(\phi=90^{\circ}\right)$ is not so critical, since the antenna is symmetric in relation to the $X_{A} Z_{A}$ plane.

\subsection{Ideal Flat Subreflector}

The first example used for validation was an ideal flat subreflector. The flat subreflector was analyzed using the aforementioned method by assuming ideal elements. Thus, the components $\rho_{x y}$ and $\rho_{y x}$ of the $\mathbf{R}$ matrix in Equation (3) were zero for each element $\left(m_{R}, n_{R}\right)$ of the reflectarray. This meant that there was no cross polarization generated on the reflectarray's elements. The moduli of $\rho_{x x}$ and $\rho_{y y}$ were also one because lossless elements were considered. In general, an ideal reflectarray introduces a phase shift for each polarization at each element $\left(m_{R}, n_{R}\right)$, and the $\mathbf{R}$ matrix for each one is

$$
\mathbf{R}\left(m_{R}, n_{R}\right)=\left\{\begin{array}{cc}
\exp \left[j \varphi_{x x}\left(m_{R}, n_{R}\right)\right] & 0 \\
0 & \exp \left[j \varphi_{y y}\left(m_{R}, n_{R}\right)\right]
\end{array}\right\} .
$$

If $\phi_{x x}$ and $\phi_{y y}$ in Equation (22) are constant throughout the reflectarray, the reflectarray produces the same phase shift at each element, and the effect obtained is the same as for a metal plane. This planar subreflector/parabolic main reflector geometry can be

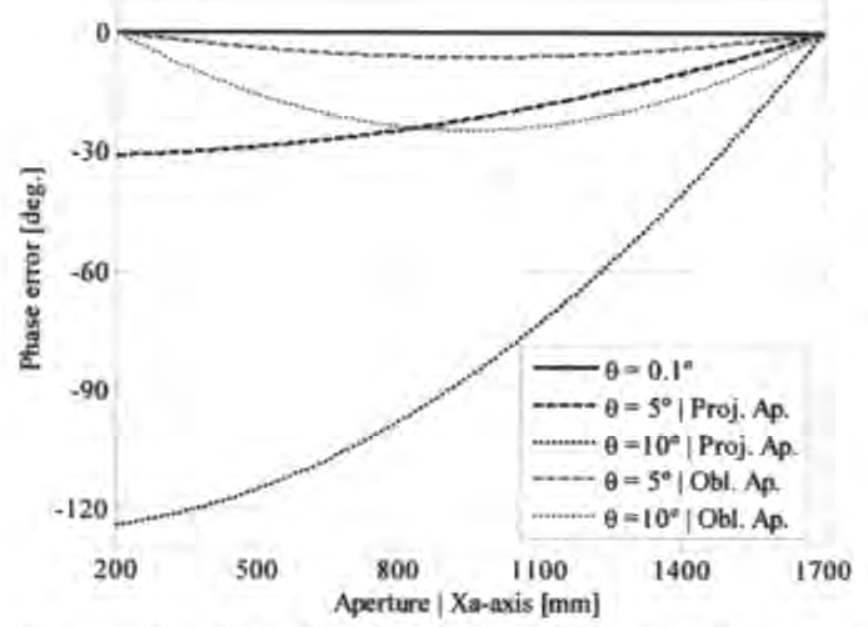

Figure 4. The vertical main cut of the phase-error map for several radiation angles and the two different apertures, $f=11.95 \mathbf{G H z}$.

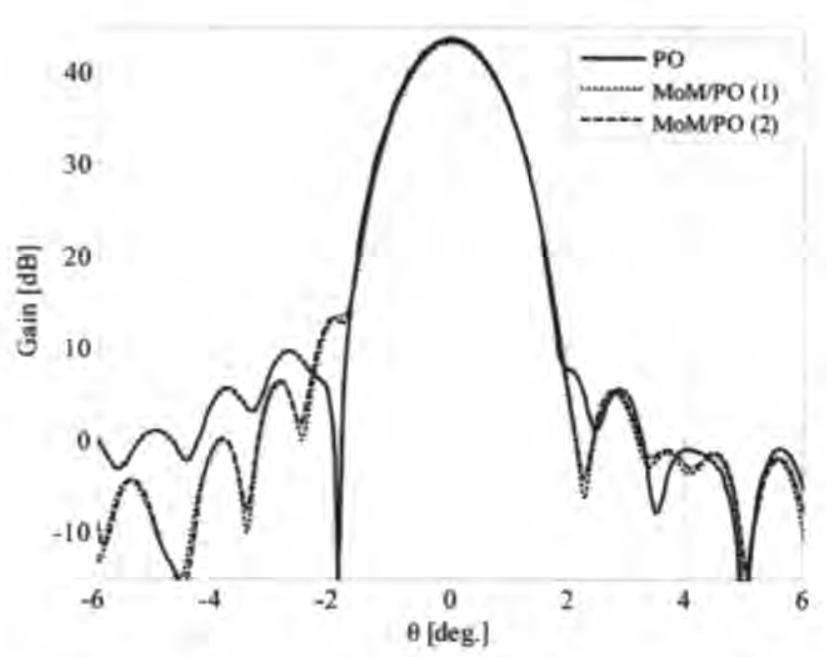

Figure 5. The elevation $\left(\phi=0^{\circ}\right)$ radiation pattern obtained with the plane-subreflector/parabolic-reflector structure. A comparison is shown of the PO analysis with GRASP8; MoM/PO (1), with projected aperture; and MoM/PO (2), with oblique aperture.

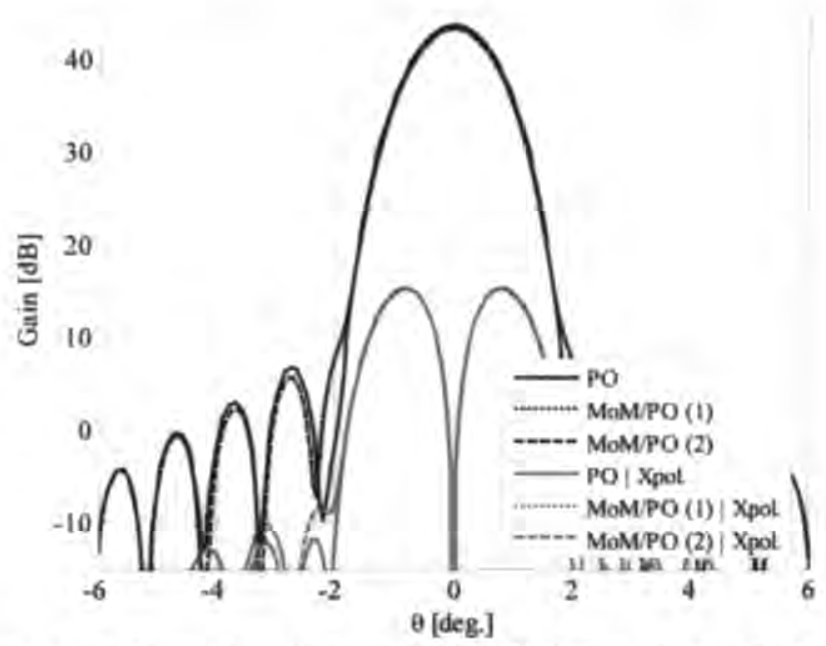

Figure 6. The azimuth $\left(\phi=90^{\circ}\right)$ radiation pattern obtained with the plane-subreflector/parabolic-reflector structure. A comparison is shown of the PO analysis with GRASP8; MoM/PO (1), with projected aperture; and MoM/PO (2), with oblique aperture.

analyzed using GRASP8 from Ticra [28], considering an ideal flat metallic plate as a subreflector. GRASP8 has been widely used for the analysis and design of reflectors, and its reliability has been proven.

The described configuration was analyzed using the MoM/PO technique for two aperture planes, as well as with GRASP8, and the results are compared in Figures 5 and 6. Very good agreement was obtained among the different simulations, including the sidelobe region, especially in azimuth. The differences between the results obtained with the projected and oblique apertures was very small, and only a slight difference in the gain was found (see Table 2) because the main lobe was in the $Z_{A}$ direction, where the phase error was zero. 
Table 2. Results for an ideal reflectarray.

\begin{tabular}{|c|c|c|c|}
\hline & $\begin{array}{c}\text { MoM/PO (1) } \\
\text { (Projected } \\
\text { aperture) }\end{array}$ & $\begin{array}{c}\text { MoM/PO (2) } \\
\text { (Oblique } \\
\text { aperture) }\end{array}$ & $\begin{array}{c}\text { PO } \\
\text { (GRASP8) }\end{array}$ \\
\hline Gain [dBi] & 43.34 & 43.41 & 43.64 \\
\hline X-pol. [dB] & -28.19 & -28.20 & -28.00 \\
\hline
\end{tabular}

The gains and cross-polarization levels of the radiation patterns for the different cases are summarized in Table 2. A difference in the gain of $0.3 \mathrm{~dB}$ was found, mainly because the model used for the primary feed was not the same for the two methods. The feed horn was modeled in GRASP8 as a Gaussian beam, and as a $\cos ^{q}(\theta)$ function in the MoM/PO tool. In both cases, the models were adjusted so that the illumination level at the edges of the subreflector was the same. However, the directivity of the two models differed by approximately $0.2 \mathrm{~dB}$, which justified the small difference in gain. In fact, this was the difference between the gain obtained in the case of the MoM/PO analysis with the oblique aperture and that obtained with GRASP8.

Concerning computational efficiency, the proposed technique took 112 seconds to analyze the antenna, using a Pentium 4 computer with a $2.4 \mathrm{GHz} \mathrm{CPU}$ and $1 \mathrm{~GB}$ of RAM, for the calculation of the three-dimensional radiation pattern for the two linear polarizations. The radiation pattern was computed in the region defined by the interval $[-0.17,0.17]$ in both in $u$ and $v$, using a regular mesh of $180 \times 180$ points. It must be noted that in this case of an ideal sub-reflectarray, the analysis using the Method of Moments (MoM) was not run. On the other hand, GRASP 8.1 took 475 seconds to analyze the antenna, considering ideal metallic reflectors and the same mesh and polarization parameters. Although the radiation pattern can be calculated in a larger interval, the selected case correspond to $0^{\circ}<\theta<10^{\circ}$ and $0^{\circ}<\phi<360^{\circ}$, which included the angular margin required for the coverage of the Earth from a geostationary satellite.

\subsection{Real Reflectarray as Subreflector}

As a second case, a real reflectarray was analyzed as the subreflector. In this case, the four components of the $\mathbf{R}$ matrix in Equation (3) were computed on each reflectarray element by the MoM assuming local periodicity. The amplitudes of $\rho_{x x}$ and $\rho_{y y}$ terms should have been slightly smaller than one, because of the dissipative losses in the reflectarray element. The terms $\rho_{x y}$ and $\rho_{y x}$ should have been different from zero, because the patches produced some cross polarization. In order to compare the results with those previously obtained for an ideal sub-reflectarray, a similar case was considered. Thus, the reflectarray should have had the same behavior as a metallic plane, that is, the phase of the reflection coefficient had to be constant throughout the reflectarray. However, in this case, the losses and cross-polar radiation introduced by the reflectarray were evaluated.

A two-layer reflectarray of stacked patches over a ground plane was defined as shown in Figure 7. Since the main potential applications of this structure are space applications, a sandwich with space-qualified materials was defined. Each array of patches was supposed to have been printed on a 0.125 mm-thick Kapton
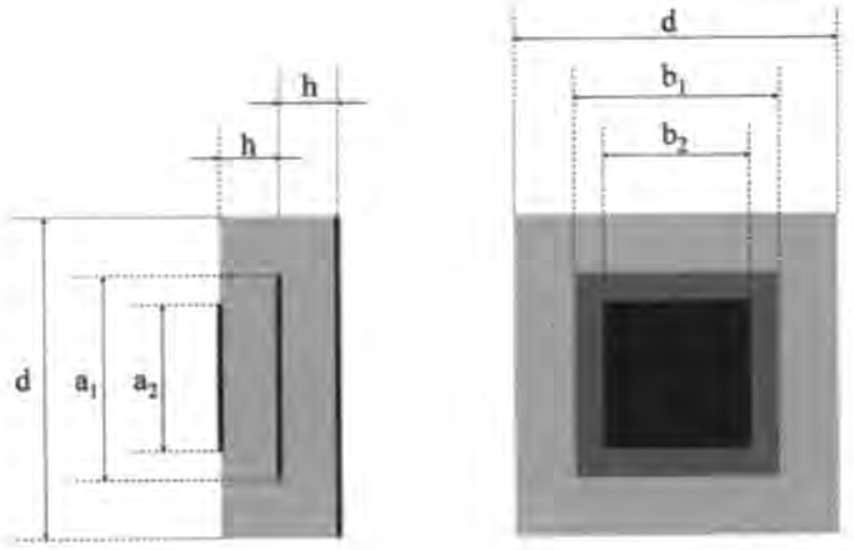

Figure 7. The sub-reflectarray periodic cell.

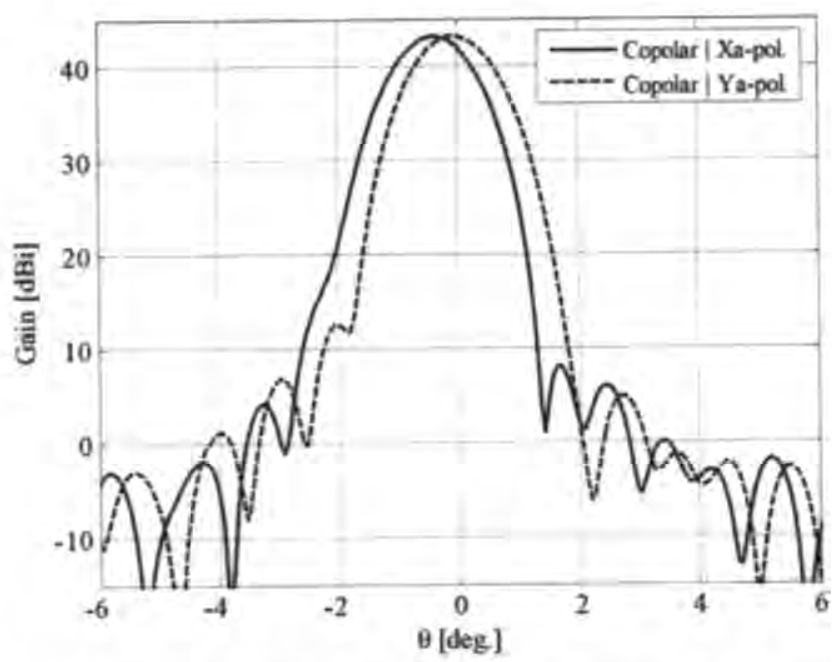

Figure 8a. The radiation pattern obtained with the real subreflectarray/parabolic-reflector structure: main cut at the $\phi=0^{\circ}$ plane.

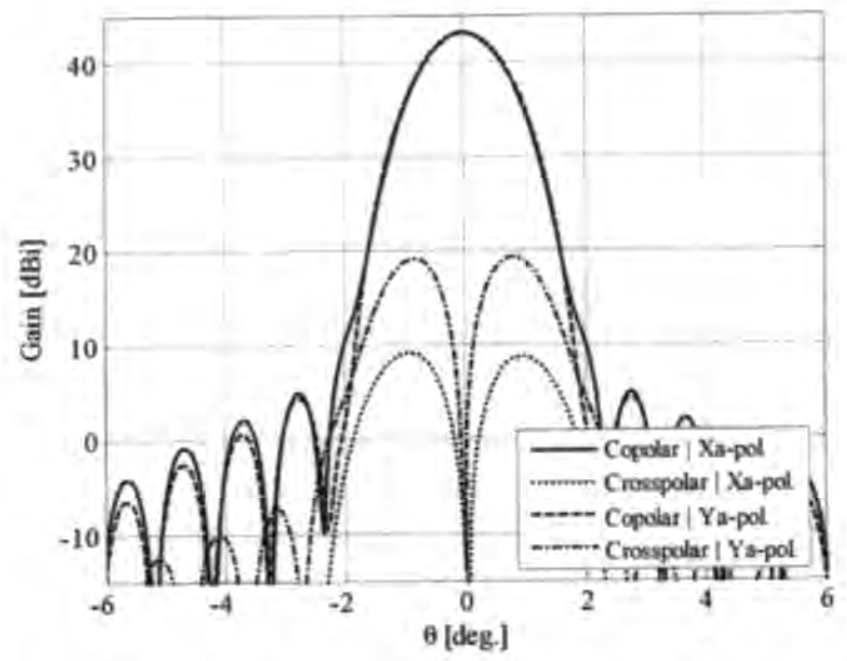

Figure $8 \mathrm{~b}$. The radiation pattern obtained with the real subreflectarray/parabolic-reflector structure: main cut at the $\phi=90^{\circ}$ plane. 
Phase distribution of the reflection coefficient | Xr-polarisation

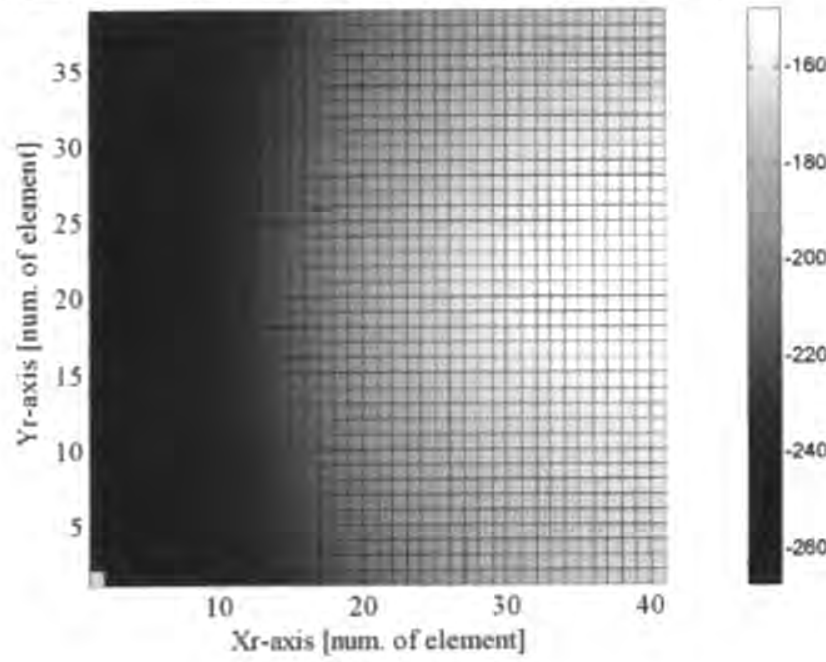

(a)

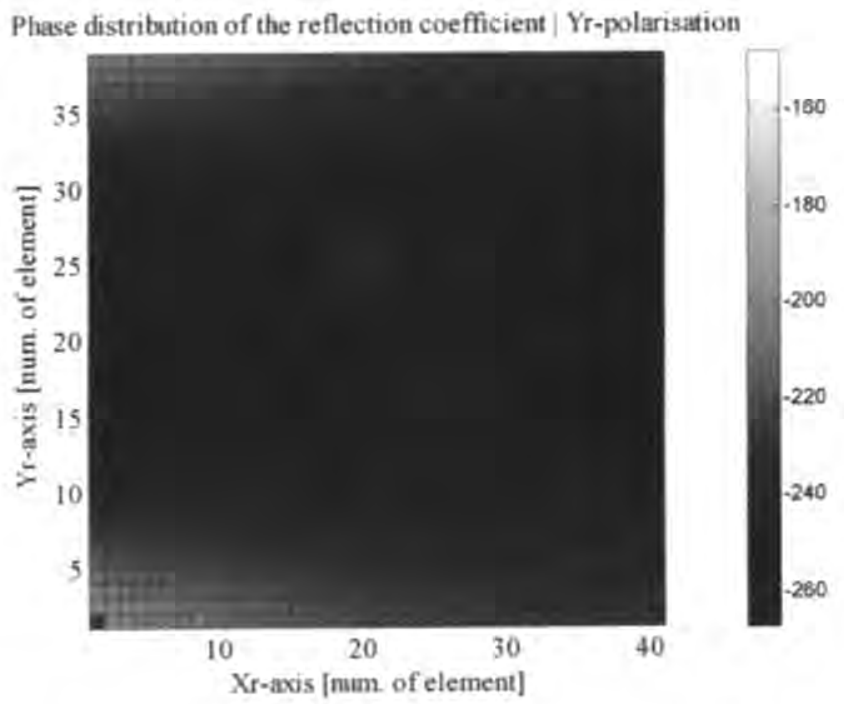

(b)

Figure 9. The phase shift produced by the sub-reflectarray for Xr polarization (a) and Yr polarization (b).

film ( $\left.\varepsilon_{r}=2.98, \tan \delta=0.005\right)$, bonded to a 0.125 mm-thick quartz fabric composite layer $\left(\varepsilon_{r}=2, \tan \delta=0.0058\right)$. The two layers and the ground plane were then separated by a $3.5 \mathrm{~mm}$-thick quartz honeycomb $\left(\varepsilon_{r}=1.046, \tan \delta=0.00076\right)$ (see Figure 7). As a first approximation, the patch dimensions for all the periodic cells of the reflectarray were the same $\left(a_{1}=b_{1}=10 \mathrm{~mm}\right.$, $a_{2}=b_{2}=7 \mathrm{~mm}$ ), so that the phase of the reflection coefficient for all the elements should have been nearly constant. The phase of the reflection coefficient varied with the angle of incidence, but the variation was usually very small for angles under $40^{\circ}$.

The calculated radiation patterns are shown in Figure 8. For the vertical polarization ( $X_{A}$ polarization), a tilt of about $-0.4^{\circ}$ was observed. This was because of the angle of incidence: the feed horn was very close to the reflectarray, and the angles of incidence on a number of elements were high. If the angle of incidence was less than $40^{\circ}$, the phase of the reflection coefficient was nearly constant. However when it became larger, the reflection coefficient changed, and the effect produced was a progressive phase along the $X_{R}$ axis. This effect is shown in Figure 9a, and it implied that in this antenna, the analysis and design of the sub-reflectarray elements had to be carried out by taking into account the real angle of incidence of the impinging wave from the primary feed.

For the other polarization ( $Y_{A}$ polarization), the phase variation was in the orthogonal direction (see Figure 9b). In this case, the $X_{A} Z_{A}$ plane was a symmetry plane, and the phase distribution was also symmetric in relation to that plane. As a result, the beam was not squinted for $Y_{A}$ polarization, as shown in Figure 8 .

The gain calculated for this configuration was $43.12 \mathrm{dBi}$ for the $X_{A}$ polarization, which implied losses of $0.22 \mathrm{~dB}$ (see Table 2). This parameter was computed by considering a projected aperture for the integration of the radiated field. However, the results were very similar by integrating at the oblique aperture. The cross-polar level for this polarization was different from that obtained with the ideal sub-reflectarray because of the cross-polar

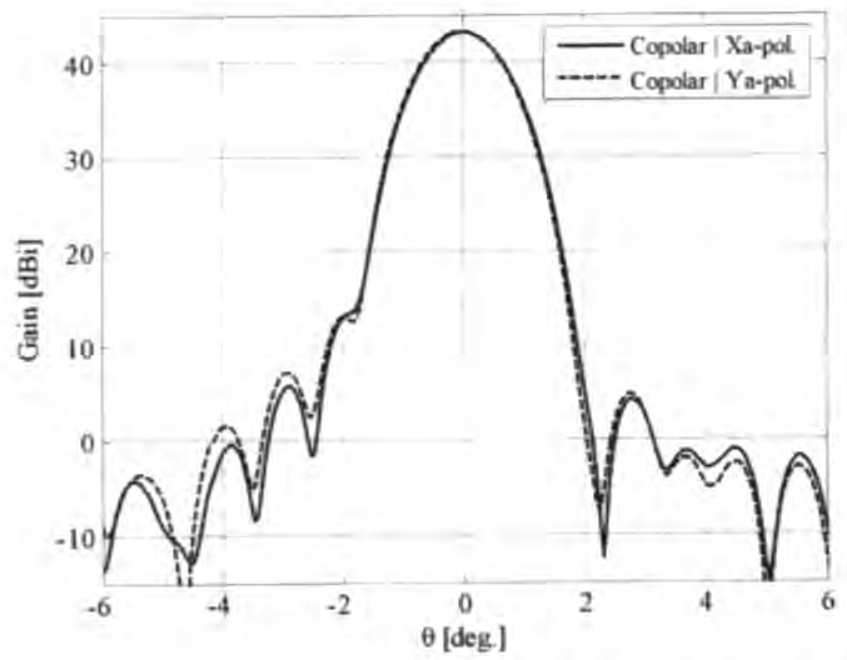

Figure 10a. The radiation pattern obtained with the designed sub-reflectarray/parabolic-reflector structure: main cut at the $\phi=0^{\circ}$ plane.

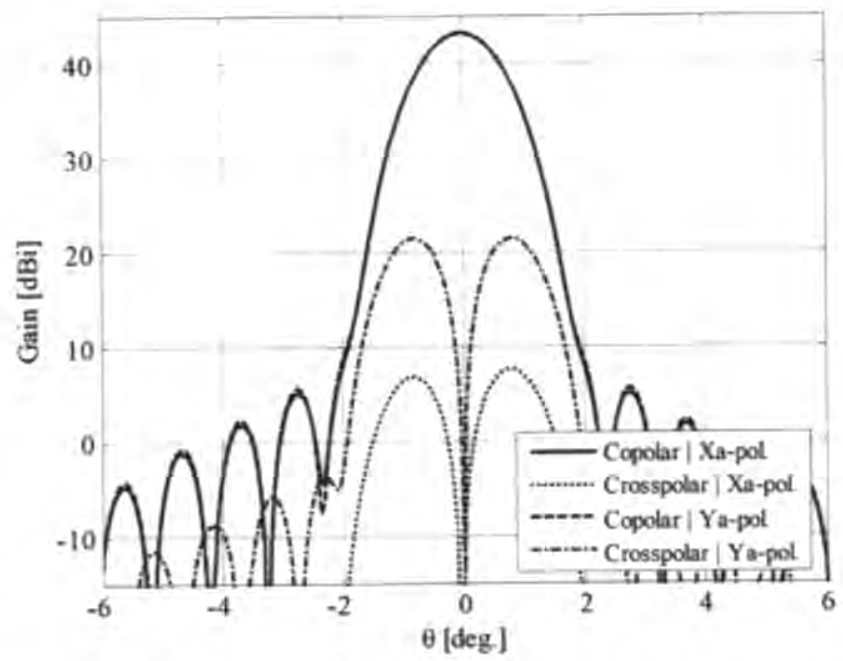

Figure $10 \mathrm{~b}$. The radiation pattern obtained with the designed sub-reflectarray/parabolic-reflector structure: main cut at the $\phi=90^{\circ}$ plane. 
component introduced by the patches. In this case, the cross-polar level differed for $X_{A}$ and $Y_{A}$ polarizations (see Figure 8), while in the case of the ideal sub-reflectarray, the cross-polar pattern was the same for the two linear polarizations as well as for a single offset reflector (see Figure 6).

The effect of the variation of the incidence angle of the impinging wave on the reflectarray cells can be compensated for through a suitable design of the reflectarray, taking into account this angle. A reflectarray was thus designed so that all the elements produced exactly the same phase shift. The design was carried out using the technique described in [4], so that a reflection coefficient with a phase of $-180^{\circ}$ was achieved. All the geometric data were the same as in the previous case, as well as the materials of the multilayer structure.

The photo-etching mask of the lower layer of the designed reflectarray was obtained. Because of the small differences caused by the incidence angles, the patch dimensions slightly vary along the $X_{r}$ axis. In fact, the difference in size between the patches

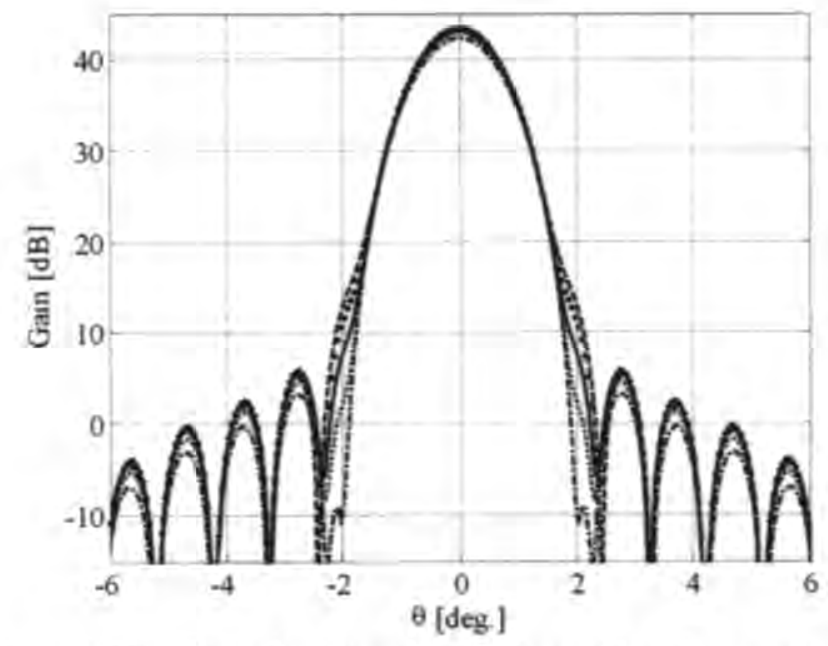

Figure 11a. The azimuth main cut for an Xa-axis scanning of the electrical beam scanning carried out by introducing a progressive phase on the reflectarray surface.

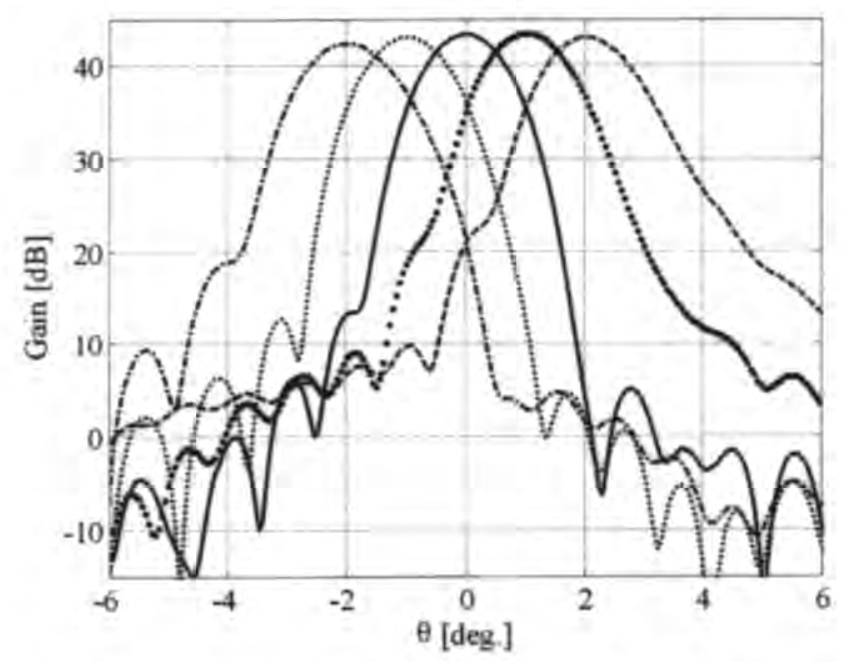

Figure 11b. The elevation main cut for an Xa-axis scanning of the electrical beam scanning carried out by introducing a progressive phase on the reflectarray surface.

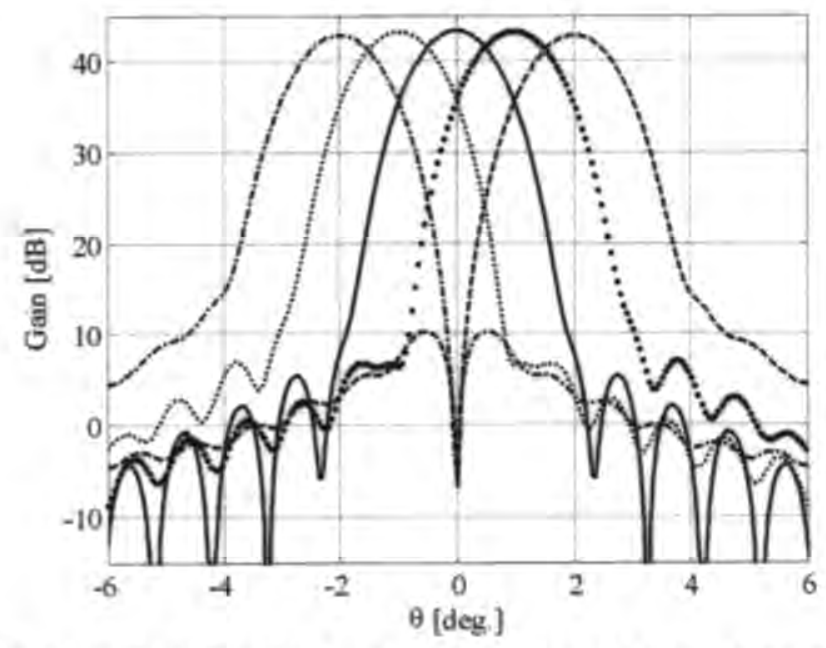

Figure 11c. The azimuth main cut for an Ya-axis scanning of the electrical beam scanning carried out by introducing a progressive phase on the reflectarray surface.

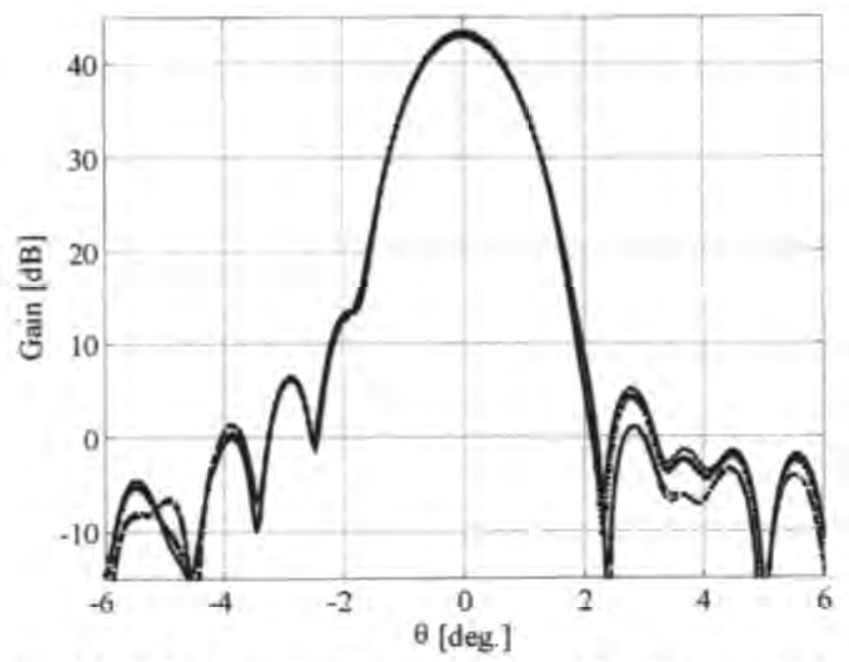

Figure 11d. The elevation main cut for an Ya-axis scanning of the electrical beam scanning carried out by introducing a progressive phase on the reflectarray surface.

under the largest and smallest incidence angle was about $2 \mathrm{~mm}$, with the size of the periodic cell having been $13 \mathrm{~mm} \times 13 \mathrm{~mm}$. The size of the patches in the upper layer was scaled from those in the lower layer by a factor of 0.7 .

The designed reflectarray was used as subreflector in the same configuration, and the whole antenna was analyzed by $\mathrm{MoM} / \mathrm{PO}$. The radiation patterns obtained are shown in Figure 10 for both the $\phi=0^{\circ}$ and $\phi=90^{\circ}$ planes. As a result of the design taking into account the angle of incidence of the impinging wave from the primary feed on each element of the reflectarray, no beam squinting was found in this case.

In this case, the computational time increased to 235 seconds because of the analysis of the real reflectarray by the MoM. However, the time consumed was still lower than the time spent by GRASP. 


\section{Application: Beam-Scanning Antenna}

The proposed configuration can be used as a high-gain beamscanning antenna. Two techniques can be used to obtain beam scanning: one based on mechanical beam scanning and the other on electronic beam scanning. On the one hand, the beam can be scanned by simply rotating the sub-reflectarray, as in classic dualreflector configurations. On the other hand, the reflectarray can be used to scan the beam by adding controllable phase-shifter elements. The phase distribution on the reflectarray can thus be modified by electronic control, and consequently the beam performance can be changed without mechanical devices. The beam scanning can also be achieved with a large reflectarray or a phased array, but in the configuration considered here, the electronically reconfigurable surface is smaller, simplifying the design and manufacture of the antenna.

If beam scanning is required in only one dimension, the reflectarray should introduce a controllable progressive phase along the scanning axis. The electronic control can be common for all the elements in a row or in a column, that is, control at the reflectarray row or column level. For example, if a progressive phase is introduced along the $X_{R}$ axis of the sub-reflectarray, an elevation scanning $\left(X_{A}\right)$ of the beam will be obtained. On the other hand, if two-dimensional beam scanning is specified, the phase-shift control has to be independent for all the sub-reflectarray elements.

The pattern performance of the geometry assuming an ideal sub-reflectarray for $X_{A}$ polarization and elevation $\left(\phi=0^{\circ}\right)$ scanning is plotted in Figures $11 \mathrm{a}$ and $11 \mathrm{~b}$, while the behavior of an azimuth $\left(\phi=90^{\circ}\right)$ scanning for the same polarization is plotted in Figures 11c and 11d. The plots show good performance for $\pm 2^{\circ}$ scanning angles, especially for azimuth. The same study was carried out for the other linear polarization ( $Y_{A}$ polarization), and similar results were obtained. The phase distribution of the reflection coefficient required for $+2^{\circ}$ beam scanning on the $X_{A}$ axis is shown in Figure 12.

The cross-polar radiation pattern depends on the shape of the reflectarray elements and, therefore, low cross-radiation elements

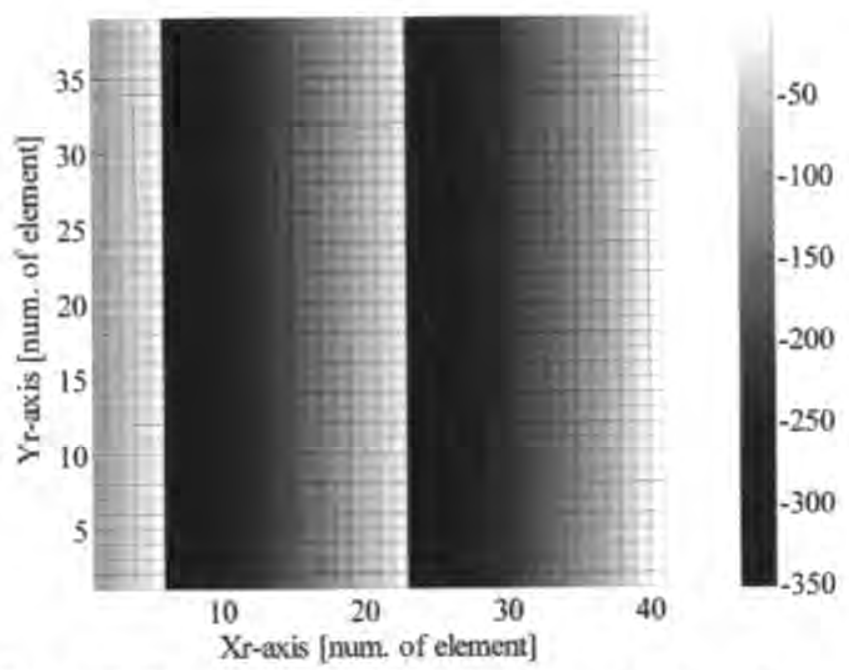

Figure 12. The progressive phase distribution of the reflection coefficient required for $+2^{\circ}$ beam scanning on the Xa axis.
Table 3. The gain performance [dBi].

\begin{tabular}{|c|c|c|c|c|c|}
\hline $\begin{array}{c}\text { Scanning } \\
\text { Angle }\end{array}$ & $-\mathbf{2}^{\circ}$ & $-\mathbf{1}^{\circ}$ & Focused & $\mathbf{1}^{\circ}$ & $\mathbf{2}^{\circ}$ \\
\hline Elevation & 42.4 & 43.0 & \multirow{2}{*}{43.3} & 43.3 & 43.0 \\
\cline { 1 - 3 } \cline { 5 - 6 } Azimuth & 42.8 & 43.2 & & 43.2 & 42.8 \\
\hline
\end{tabular}

$$
0.06
$$

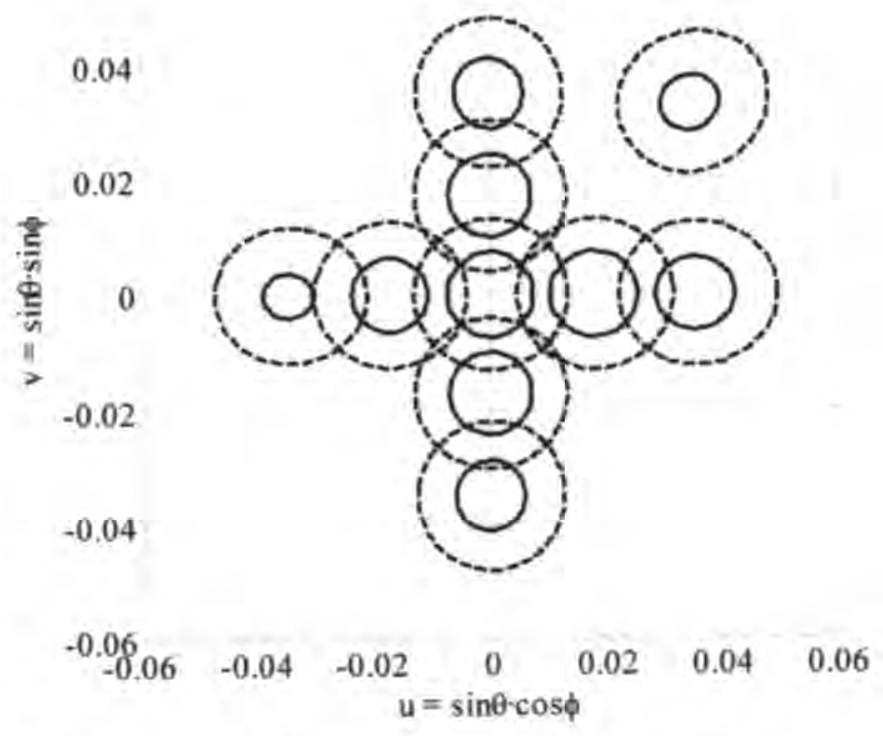

Figure 13. Two-dimensional electrical beam scanning. For each beam, gain levels of $42 \mathrm{dBi}$ (continuous lines) and $39 \mathrm{dBi}$ (dashed lines) have been plotted.

should be used. In the case of the ideal phase shifters considered here, the cross polarization for the overall antenna is slightly increased when the beam is defocused. However, the cross-polar level was of the order of $-30 \mathrm{~dB}$ below the co-polar gain for the scanning angles considered in this work.

As the reduction of gain is an important parameter in beamscanning antennas, the gain was computed for a beam scanning from $-2^{\circ}$ to $+2^{\circ}$, and the results are summarized in Table 3 . The dimensional beam scanning is possible with this antenna configuration, if the phase control is implemented at element level. The results for two-dimensional scanning are shown in Figure 13.

The beam-scanning performance presented here is based on an ideal sub-reflectarray, and does not depend on the geometry of the phase-shifter element. For printed-patch reflectarrays, the phase of the reflection coefficient can be modified by varying the $\varepsilon_{r}$ of the substrate, which can be achieved with liquid-crystal technology. If liquid crystal is used as a substrate, the $\varepsilon_{r}$ can be modified by applying an external voltage, and the phase of the reflection coefficient of the reflectarray cell varies [20]. If a different voltage is applied to each row or column of the reflectarray, a progressive phase can be obtained for the reflection coefficient, and beam scanning is achieved.

Other reflectarray elements, such as patches aperture coupled to delay lines, can be used to implement the phase control by inserting varactors, PIN diodes, or MEMS (micro-electromechanical switches) [29-31]. These technologies are suitable for control at the element level, which permits two-dimensional beam scanning. 


\section{Conclusion}

A novel modular technique, based on the Method of Moments and Physical Optics (MoM/PO), for the analysis of dualreflector antennas with a reflectarray as the subreflector has been presented. The analysis approach combines two different techniques: basically, MoM for the analysis of the reflectarray and PO for the reflector. A simplification is made in PO that allows a significant reduction in the computational time, at the cost of a small phase error in the calculation of the field at the main aperture. Two apertures have been considered, and the phase error introduced has been evaluated for the two cases. The technique has been applied to the analysis of a focused-beam antenna with a planar metallic subreflector. The results have been compared with those obtained with GRASP8, validating the technique. The case of a real reflectarray as a subreflector has also been studied by designing a reflectarray that corrects the effect of the angle of incidence on the reflectarray's surface. The geometry can be used to scan the beam by introducing an appropriate progressive phase on the reflectarray's surface, achieved by inserting electronically controllable elements. Assuming an ideal sub-reflectarray, the beam-scanning behavior has been studied and the results showed good performance. MoM/PO can be applied in a pattern synthesis process because it is time-efficient and accurate, providing good accuracy in the prediction of sidelobes.

\section{Acknowledgment}

This work was supported by the European Space Agency (ESA/ESTEC) under contract ESTEC/19571/06/NL/JA, and by the Spanish Commission for Science and Technology (CICYT) under contract TEC2007-63650/TCM.

\section{References}

1. J. Huang, "Printed Reflectarray Antenna: History, Design and Application," in Antonio Tazón (ed.), Advances on Antennas, Reflectors and Beam Control, Kerala, India, Research Signpost, 2005, pp. 1-24.

2. J. A. Encinar, E. Carrasco and M. Arrebola, "Analysis, Design and Applications of Multi-Layer Printed Reflectarrays," in Antonio Tazón (ed.), Advances on Antennas, Reflectors and Beam Control, Kerala, India, Research Signpost, 2005, pp. 25-51.

3. D. M. Pozar, S. D. Targonski and R. Pokuls, "A Shaped-Beam Microstrip Patch Reflectarray," IEEE Transactions on Antennas and Propagation, AP-47, 7, July 1999, pp. 1167-1173.

4. J. A. Encinar, "Design of Two-Layer Printed Reflectarrays Using Patches of Variable Size," IEEE Transactions on Antennas and Propagation, AP-49, 10, October 2001, pp. 1403-1410.

5. J. A. Encinar, L. Sh. Datashvili, J. A. Zornoza, M. Arrebola, M. Sierra-Castañer, J. L. Besada-Sanmartín, H. Baier and H. Legay, "Dual-Polarization Dual-Coverage Reflectarray for Space Applications," IEEE Transactions on Antennas and Propagation, AP54, 10, October 2006, pp. 2827-2837.
6. M. Y. Ismail, W. Hu, J. A. Encinar, R. Cahill, R. Dickie, H. S. Gamble, V. F. Fusco, D. Linton, S. P. Rea and N. Grant, "Liquid Crystal Based Reflectarray Antenna with Switchable Sum and Difference Patterns," 29th ESA Antenna Workshop on Multiple Beams and Reconfigurable Antennas, ESA-ESTEC, Noordwijk, The Netherlands, April 18-20, 2007.

7. M. E. Bialkowski, A. W. Robinson and H. J. Song, "Design, Development and Testing of X-Band Amplifying Reflectarrays," IEEE Transactions on Antennas and Propagation, AP-50, 8, August 2002, pp. 1065-1076.

8. D. Pilz and W. Menzel, "Folded Reflectarray Antenna," Electronics Letters, 34, 9, February 2007, pp. 832-833.

9. D. M. Pozar, "Bandwidth of Reflectarrays," Electronics Letters, 39, 21, October 2003, pp. 1490-1491.

10. J. Huang, "Bandwidth Study of Microstrip Reflectarray and a Novel Phased Reflectarray Concept," in IEEE International Symposium on Antennas and Propagation, Newport Beach, CA, USA, June 18-23, 1995.

11. J. A. Encinar and J. A. Zornoza, "Three-Layer Printed Reflectarrays for Contoured Beam Space Applications," IEEE Transactions on Antennas and Propagation, AP-52, 5, May 2004, pp. 1138-1148.

12. E. Carrasco, M. Barba and J. A. Encinar, "Reflectarray Element Based on Aperture-Coupled Patches with Slots and Lines of Variable Length," IEEE Transactions on Antennas and Propagation, AP-55, 3, March 2007, pp. 820-825.

13. D. M. Pozar, "Wideband Reflectarrays Using Artificial Impedance Surfaces," Electronics Letters, 43, 3, February 2007.

14. A. W. Rudge and N. A. Adatia, "Offset-Parabolic-Reflector Antennas: A Review," Proc. IEEE, 66, 12, December 1978, pp. 1592-1623.

15. B. Khayatian, Y. Rahmat-Samii, and J. Huang, "Radiation Characteristics of Reflectarray Antennas: Methodology and Applications to Dual Configurations," First European Conference on Antennas and Propagation - EuCAP 2006, Nice, France, November 6-10, 2006.

16. R. Leberer and W. Menzel, "A Dual Planar Reflectarray with Synthesized Phase and Amplitude Distribution," IEEE Transactions on Antennas and Propagation, AP-53, 11, November 2005, pp. 3534-3539.

17. S. Xu, H. Rjagopalan, Y. Rahmat-Samii and W. Imbriale, "A Novel Reflector Surface Distortion Compensating Technique Using a Sub-Reflectarray," IEEE International Symposium on Antennas and Propagation, Honolulu, HI, USA, June 10-15, 2007.

18. H. M. Braun, P. E. Knobloch, "SAR on Small Satellites Shown on the SAR-Lupe Example," Proceedings of the International Radar Symposium 2007 (IRS 2007), Cologne, Germany, September 5-7, 2007

19. R. J. Martin and D. H. Martin, "Quasi-Optical Antennas for Radiometric Remote-Sensing," IEE Electron. \& Comm. Eng. J., 8, February 1996, pp. 37-48. 
20. W. Hu, M. Y. Ismail, R. Cahill, H. S. Gamble, R. Dickie, V. F. Fusco, D. Linton, S. P. Rea and N. Grant, "Phase Control of Reflectarray Patches Using Liquid Crystal Substrate," First European Conference on Antennas and Propagation - EuCAP 2006, Nice, France, November 6-10, 2006.

21. A. Moessinger, R. Marin, S. Mueller, J. Freese and R. Jackoby, "Electronically Reconfigurable Reflectarrays with Nematic Liquid Crystals," Electronic Letters, 42, 16, August 2006, pp. 899-900.

22. W. Hu et al., "Liquid Crystal Tunable mm Wave Frequency Selective Surface," IEEE Microwave and Wireless Components Letters, 17, 9, September 2007, pp. 667-669.

23. M. Arrebola, Y. Álvarez, J. A. Encinar and F. Las-Heras, "Accurate Analysis of Printed Reflectarrays Considering the Near Field of the Primary Feed," accepted for publication in IET Microw. Antennas Propag., 2008.

24. M. Arrebola, J. A. Encinar, Y. Álvarez, F. Las-Heras and W. Menzel, "Design of a Reflectarray with Three Shaped Beams Using the Near-Field Radiated by the Feeds," First European Conference on Antennas and Propagation - EuCAP 2006, Nice, France, November 6-10, 2006.

25. C. Wan and J. A. Encinar, "Efficient Computation of Generalized Scattering Matrix for Analyzing Multilayered Periodic Structure," IEEE Transactions on Antennas and Propagation, AP-43, 11, November 1995, pp.1233-1242.

26. J. F. Kauffman, W. F. Croswell and L. J. Jowers, "Analysis of the Radiation Patterns of Reflector Antennas," IEEE Transactions on Antennas and Propagation, AP-24, 1, January 1976, pp. 53-65.

27. M. Arrebola, J. A. Encinar and M. Barba, "Multi-Fed Printed Reflectarray with Three Simultaneous Shaped Beams for LMDS Central Station Antenna," IEEE Transactions on Antennas and Propagation, AP-56, 6, June 2008, pp. 1518-1527.

28. F. Jensen, Reference Manual for GRASP8. GRASP8/GRASP8W Version 8.2.5, Copenhagen, Denmark, TICRA Engineering Consultants, March 2002.

29. M. Barba, E. Carrasco, J. E. Page and J. A. Encinar, "Electronic Controllable Reflectarray Elements in X Band," FREQUENZ - Journal of RF-Engineering and Telecommunications, October 2007.

30. C. Apert, T. Koleck, P. Dumon, T. Dousset and C. Renard, "ERASP: A New Reflectarray Antenna for Space Applications," First European Conference on Antennas and Propagation - EuCAP 2006, Nice, France, November 6-10, 2006.

31. R. Sorrentino, "MEMS-Based Reconfigurable Reflectarrays," 2nd European Conference on Antennas and Propagation - EuCAP 2007, Edinburgh, UK, November 12-16, 2007. (A6) 